\title{
Radio-over-fibre for green wireless access networks
}

\author{
Trevor J. Hall • Frédéric Lucarz • John Mitchell • Patrice Pajusco
}

Published online: 21 December 2012

(C) Institut Mines-Télécom and Springer-Verlag France 2012

The genesis of this special issue was the International Symposium on Green Radio over-fibre and all-Optical Wireless technologies for Access Networks: GROWAN 2011, 15 17 June 2011, Brest, France. A selection of international key speakers from both industry and academia were invited to address radio-over-fibre and all-optical wireless technologies with a focus on minimising overall energy consumption in wireless access networks. This capstone event marked the end of the tenure by Dr. Hall of the first SISCom International Chair. The Chair supported Dr. Hall while on sabbatical leave from the University of Ottawa hosted by Télécom Bretagne, Brest, France to work on 'Green Radioover-Fibre Architectures'. SISCom is a new research cluster for Information and Communications Technologies that has been established in Brittany, France, involving l'Université Européenne de Bretagne, the National Research Institute in Computer Science and Control, Télécom Bretagne and CNRS. As part of its programme for research excellence and international outreach, SISCom has launched this series of visiting chair positions for sabbatical appointments and collaborative research supervision, which will offer major potential to develop new international collaborations.

Whereas the GROWAN 2011 participants clearly defined their research as radio-over-fibre, many did not appreciate at first the relationship of their research to 'green' technology.

\section{T. J. Hall}

University of Ottawa, 800 King Edward Avenue, Ottawa, ON K1N 6N5, Canada

F. Lucarz $(\bowtie) \cdot$ P. Pajusco

Télécom Bretagne, Technopôle Brest-Iroise, CS 83818, 29238, Brest Cedex 3, France

e-mail: frederic.lucarz@telecom-bretagne.eu

J. Mitchell

University College London, Torrington Place,

London WC1E 7JE, UK
A first important outcome of the SISCom chair research was the identification of distributed antenna systems supported by an optical fibre network (or by an optical wireless network in benign environments), i.e. the radio-over-fibre (RoF) paradigm, as the most promising wireless access technology in cluttered urban environments in terms of energy consumption. Radio-over-fibre is therefore potentially a 'green' alternative to conventional approaches to wireless access. For at least two decades, RoF has been synonymous with radio frequency (RF) transport over analogue intensity-modulated direct detection (IM-DD) optical fibre links justified in terms of cost reductions. Analogue optical transport is plagued by linearity and noise issues and the industry preference is for digital solutions. A second important outcome of the SISCom chair research was that the best transport mechanism remained an open question and both digital IM-DD and digital coherent optical approaches showed promise.

In this special issue, a joint paper between the Télécom Bretagne and University of Ottawa teams: Trevor J. Hall et al. 'Radio-over-fibre access for sustainable digital cities', first introduces the vision of the sustainable 'digital city' of the third millennium that is founded on ubiquitous wireless and computing power and explores the first steps in how engineers might be able to implement this vision in respect of wireless access. The radio-over-fibre (RoF) paradigm is then identified as the most effective wireless access technology in terms of energy consumption. Moreover, digital coherent optical approaches are identified as having excellent potential.

Continuing in this spirit, the paper by Yizhuo Yang et al. 'Digitized RF-over-fiber as a cost-effective and energyefficient backhaul option for wireless communications' describes an architecture that uses links consisting of an analogue to digital convertor connected by a digital IMDD optical link to a digital to analogue convertor to digitise 
and transport RF-signals. Band pass sampling enables up and down conversion without conventional mixers. Moreover, the paper provides an analysis of the energy consumption of the architecture in comparison with the orthodox approach.

Béatrice Cabon et al. 'Comparisons of system architectures for microwave-photonics transmissions at $60 \mathrm{GHz}$ ' then compares different analogue RoF architectures that exploit photonic heterodyning or microwave photonics means of generating millimetre-wave wireless signals. Low-error transmission using at data rates up to $4.2 \mathrm{~Gb} / \mathrm{s}$ is experimentally demonstrated.

Physical layer issues are then addressed by the remaining papers. Sawsan Abdul-Majid et al. ' $90^{\circ}$ SOI optical hybrid for Radio-over-Fibre links' describes how the optical front end of a digital coherent optical receiver may be realised as a low-cost silicon photonics integrated circuit. Hamdam Nikkhah et al. 'Beam steering for wireless optical links based on an optical phased array in silicon' describe how the same integration platform may also be used to form phased array antennas for optical wireless. This provides a vision of wireless access beyond current on-off-keyed systems where advantage is taken of coherent optical transceivers equipped with 'smart antennas' that enable multiple in multiple out channels using advanced modulation formats to achieve high bit rate energy-efficient communications.

The issue concludes with three papers that consider the impact of optoelectronic device interfaces between the optical and the microwave domains on the RoF link performance: Luis Manuel Pessoa et al. 'Experimental evaluation of a R-EAM and noise impact analysis for UWB and Wi-Fi transmission in RoF networks'; Anne-Laure Billabert et al. 'Comparison of theoretical and measured $\mathrm{P} 1 \mathrm{~dB}$ of a photonic microwave link: influence of some physical parameters of the DFB laser', and Ricardo Marques Ribeiro et al. 'Optimisation of a device for pick-up of low-frequency radio-signals and transmission over polymer optical fibres'. 